\title{
CARDIOVASCULAR DISEASE RISK FACTORS IN PATIENTS WITH POSTTRAUMATIC STRESS DISORDER (PTSD): A NARRATIVE REVIEW
}

\author{
Marina Šagud ${ }^{1}$, Nenad Jakšić ${ }^{2}$, Bjanka Vuksan-Ćusa ${ }^{1,3}$, Mladen Lončar ${ }^{1}$, Ivana Lončar ${ }^{2}$, \\ Alma Mihaljević Peleš ${ }^{1}$, Davor Miličić ${ }^{4} \&$ Miro Jakovljević ${ }^{1}$ \\ ${ }^{I}$ School of Medicine, University of Zagreb, Department of Psychiatry, \\ University Hospital Center Zagreb, Zagreb, Croatia \\ ${ }^{2}$ National Center for Psychotrauma, Department of Psychiatry, University Hospital Center Zagreb, Zagreb, Croatia \\ ${ }^{3}$ School of Medicine, University of Osijek, Osijek, Croatia \\ ${ }^{4}$ School of Medicine, University of Zagreb, Department for Cardiovascular Diseases, \\ University Hospital Centre Zagreb, Zagreb, Croatia
}

received: 15.7.2017;

revised: 21.9.2017;

accepted: 5.10 .2017

\begin{abstract}
SUMMARY
Posttraumatic stress disorder (PTSD) is a chronic condition related to severe stress and trauma. There is a mounting evidence about increased prevalence and mortality from cardiovascular diseases (CVD) in patients with PTSD. This review summarizes the current data on possible relations between PTSD and increased risks of CVD, including biological, psychological and behavioral factors. Biological factors refer to increased prevalence of metabolic syndrome (MetS), hypertension, elevation of pro-inflammatory cytokines and homocysteine levels. Peripheral Brain-derived neurotropic factor (BDNF), serum N-terminal pro-brain natriuretic peptide (NT-proBNP) and quantitative electroencephalogram (qEEG) are promising surrogate markers of increased cardiovascular risk. Among psychological factors, some personality traits, such as neuroticism and trait impulsivity/hostility, contribute to the development of PTSD, and are associated with general cardiovascular distress. Recently, type-D (distressed) personality is usually investigated in relation to cardiovascular morbidity, but in populations other than PTSD patients. Behavioral factors refer to unhealthy life-styles, encompassing high smoking rate, drug substances abuse and addiction, physical inactivity and unhealthy diet. The relationships among all these factors are complex and yet incompletely taken into consideration. Because of a high prevalence of CVD in patients with PTSD, there is a strong need for a more intensive focus on this vulnerable population in both primary and secondary cardiovascular prevention as well as in effective treatment possibilities.
\end{abstract}

Key words: PTSD - cardiovascular disease (CVD) - CVD risk factors - personality - type-D personality- resilience - resilience enhancing strategies

\section{INTRODUCTION}

Experiencing severe stress during traumatic events may lead to posttraumatic stress disorder (PTSD). It is a prevalent, chronic, and often disabling state, characterized by re-experiencing, avoidance, and hyperarousal symptoms. It has significant impact on public health, including high levels of comorbidity with other psychiatric disorders, e.g., mood and anxiety disorders, substance abuse, personality disorders (Goldstein et al. 2016).

Lifetime prevalence of PTSD in general population is reported to be from $1.8 \%$ in China (Guo et al. 2016) up to $6.1 \%$ in the United States (Goldstein et al. 2016, Kessler et al. 2005). Development of PTSD is highly complex and multifactorial, and is caused by a combination of individual vulnerability (e.g., genetics, childhood experiences, personality traits), social-ecological factors (e.g., post-deployment family and social support) and the extent of the intensity, chronicity and number of traumatic events (DiGangi et al. 2013, Jakovljević et al. 2012). War is one of the most severe stressors inhuman life, and the prevalence of PTSD among active duty military personnel and war veterans is particularly high (Gates et al. 2012). For example, after the Homeland War in Croatia, the number of persons diagnosed with war-related PTSD was 32,000, with some research suggesting around $18 \%$ prevalence of PTSD among individuals affected by the Croatian war (Priebe et al. 2010), and up to 33\% among exYugoslavia war refugees (Bogic et al. 2012).

In addition to numerous psychopathological and societal consequences of PTSD, this disorder increases the possibility of developing somatic illnesses, namely musculoskeletal, gastrointestinal, dermatological, pulmonary, metabolic, and, most notably, cardiovascular diseases (CVD) (Britvić et al. 2015, Pacella et al. 2013). Given these serious implications, the aim of this paper is to provide a comprehensive overview of the up-to-date research of the associations between PTSD and various biological, psychological and behavioral CVD risk factors. Thus, we would like to promote a need for an integrative clinical approach and treatment of PTSD (Jakovljević et al. 2012) and stimulate some further research in this challenging field. 


\section{POSTTRAUMATIC STRESS DISORDER: EMERGING RISK FACTORS FOR CARDIOVASCULAR DISEASE AND HIGHER MORTALITY}

Patients with PTSD have a higher mortality from multiple causes compared with normal population (Boscarino 2006), particularly from heart disease (Boscarino 2008). American war veterans with PTSD, 55 years and older, had increased risk for CVD generally, and in particular myocardial infarction, and peripheral vascular disease as well as congestive heart failure, compared to veterans without PTSD (Beristianos et al. 2016). Similarly, in a sample of 501 Croatian war veterans suffering from PTSD, aged between 36 and 65 years, the incidence of arterial hypertension, cardiac arrhythmias and myocardial infarction was significantly higher compared to a healthy control group (Britvić et al. 2015). It is now well-established that PTSD is independently associated with increased risk for coronary artery disease and related mortality (Edmondson \& Cohen 2013, Edmondson et al. 2013). Moreover, among veterans treated in an outpatient clinic, those with PTSD had worse indicators of endothelial function (Grenon et al. 2016), which might have contributed to the early onset and progression of CVD in this population. Similarly, PTSD within the Danish general population was associated with higher rates of myocardial infarction, ischaemic stroke and venous thromboembolism, irrespective of gender (Gradus et al. 2015). Finally, PTSD in war veterans was associated with ischemic changes on exercise treadmill tests (Turner et al. 2013).

The link between PTSD and increased CVD risk appears to be complex and incompletely understood, but might be related to various and interconnected biological, psychological and behavioral factors associated with PTSD.

\section{Biological factors}

Biological factors involve high blood pressure, metabolic syndrome (MetS), and chronic sympathetic hyperactivity which leads to hypothalamic-pituitaryadrenal axis (HPA) dysregulation, elevation of proinflammatory cytokines and C-reactive protein (CRP) (Kibler et al. 2014), while increased homocysteine and decreased brain-derived neurotrophic factor (BDNF) levels might also play a significant role. There are many other potential pathophysiological pathways that are reflecting in appropriate biological adaptation on chronic stress which is inherent to the PTSD. Cardiovascular side effects of psychopharmacologic treatment (see Potočnjak et al. 2016) may also increase cardiovascular disease risk in patients with PTSD.

\section{PTSD, MetS and lipid levels}

It is well-known that MetS and its components are risk factors for CVD. A recent meta-analysis reported that subjects with PTSD had an almost double increased risk for MetS compared to general population (Rosenbaum et al. 2015). This finding was consistent across different geographical regions, socio-economical settings or populations (Rosenbaum et al. 2015).

War veterans with higher severity of PTSD symptoms were more likely to meet diagnostic criteria for MetS (Heppner et al. 2009). Hypertension was the most frequent component of MetS in veterans with PTSD, with the prevalence of alarmingly high 77\% (Rosenbaum et al. 2015), which was significantly higher for an average prevalence of hypertension in general population of about $40 \%$. In civilian subjects with PTSD, severity of cardiovascular symptoms was positively correlated with body mass index (BMI), waist circumference, fibrinogen, and leptin values, and negatively correlated with insulin sensitivity (Farr et al. 2015).

Unlike in other many populations of war veterans, studies on CVD risk factors in Croatian war veterans are limited. A study on 69 treatment-resistant Croatian war veterans with PTSD has reported that those patients may be more prone to increased LDL-C concentrations, compared to healthy controls (Jergović et al. 2015). A small study has revealed increased platelet aggregation in Croatian war veterans (Vidović et al. 2011). Transcranial doppler sonography (TCD) in war sufferers with PTSD revealed a greater number of hemodynamic changes compared to a control group, mostly due to vasospasms (Kadojić et al. 1999). In addition, 30 middleaged war veterans with current PTSD had shorter peripheral blood mononuclear cells telomere length compared to age-matched healthy controls (Jergović et al. 2014). One hundred and three Croatian war veterans with PTSD had higher concentrations of cholesterol, LDL-cholesterol, triglycerides, and lower HDL-cholesterol, and thus a higher arteriosclerosis index compared to patients with depression (Solter et al. 2002).

\section{PTSD and CRP}

In the most recent meta-analysis of 12 studies, higher CRP at baseline was associated with greater risk of all-cause mortality and CVD mortality (Barron et al. 2015). Several studies reported an association between PTSD and elevated CRP levels (Canetti et al. 2014, Farr et al. 2015), particularly with re-experiencing CVD and denying symptoms (Canetti et al. 2014), and a correlation with CVD manifestations with overall PTSD severity (Farr et al. 2015, Michopoulos et al. 2015).

\section{PTSD and N-terminal pro-brain natriuretic peptide (NT-proBNP)}

Serum N-terminal pro-brain natriuretic peptide (NTproBNP) is a sensitive marker of myocardial dysfunction. While normal levels of NT-proBNP exclude significant structural and functional heart disease, most subjects with levels above normal, and almost all with values over four times the normal, do have significant myocardial dysfunction (Galasko et al. 2005). Among neuropsychiatric disorders, NT-proBNP levels were investigated in dementia. Higher NT-proBNP levels 
were accompanying dementia, both vascular (Mirza et al. 2016) and Alzheimer type (Marksteiner et al. 2014, Mirza et al. 2016). Higher NT-proBNP levels have been found also in patients with mild cognitive impairment (Marksteiner et al. 2014). However, there is no data onNT-proBNP levels in patents with other psychiatric disorders, including PTSD. Given the high incidence of different heart diseases and CV risk factors in subjects with PTSD, measuring serum NT-proBNP levels in this vulnerable population might help diagnose subclinical myocardial disease that could lead to the heart failure or damask dyspnea and exercise intolerance as a consequence of a damaged myocardium.

\section{PTSD and homocysteine}

Higher homocysteine concentrations in plasma are associated with increased $\mathrm{CV}$ mortality risk in various populations (Barron et al. 2015). For example, schizophrenia and bipolar disorder patients with hyperhomocysteinemia had 8.4 times higher chance to suffer from metabolic syndrome (MetS) compared to patients with normal homocysteine levels (Vuksan-Ćusa et al. 2011).

Increased homocysteine levels in patients with PTSD were consistently found in three cross-sectional studies with small sample sizes $(n=28-66)$. Mixed sample of male and female PTSD patients revealed elevated homocysteine levels compared to controls, in spite of normal levels of folate, vitamin B6 and B12 (de Vries et al. 2015). Those high levels could not have been explained by comorbid depression. Increased homocysteine levels were detected in non-smoking PTSD patients in a study on 66 Croatian war veterans, while there were no differences in serum lipid levels between war veterans and controls (Jendričko et al. 2009). In addition, increased serum homocysteine levels were also reported in 28 male patients with PTSD (Levine et al. 2008). In this study, the duration of PTSD symptoms predicted serum homocysteine levels (Levine et al. 2008).

\section{PTSD and proinflammatory cytokines}

Peripheral and chronic low-grade inflammation reflected by elevated circulating levels of proinflammatory cytokines might also contribute to increased CVD risk. High levels of interleukin 6 (IL-6), interleukin $1 \beta$ (IL-1 $\beta)$ and tumor necrosis factor- $\alpha$ (TNF- $\alpha$ ), along with $\mathrm{CRP}$, are associated with increased risk of morbidity and mortality in the older subjects (Michaud et al. 2013). Proinflammatory cytokines are involved in platelet activation and endothelial dysfunction (Michaud et al. 2013). Particularly IL-1 $\beta$ and IL-6 are considered to be involved in a development and acceleration of atherosclerosis (Loppnow et al. 2011).

A significant relationship between PTSD and immune dysfunction has been suggested (Kibler et al. 2014). A recent meta-analysis of inflammatory markers between patients with PTSD and healthy controls has found higher IL-6, IL-1 $\beta$, and interferon $\gamma$ levels in the PTSD group, although there was a large amount of heterogeneity among studies (Passos et al. 2015). In addition, PTSD duration was positively associated with IL-1 $\beta$ levels and severity was related to IL-6 (Passos et al. 2015).

On the contrary, 69 Croatian veterans with PTSD did not show alterations in circulating levels of CRP or the following pro-inflammatory cytokines: IL-1 $\beta$, TNF$\alpha$ and IL-6, in comparison to healthy controls (Jergović et al. 2015). However, in this study the levels of TNF- $\alpha$ and IL- 6 were measured by Multiplex Assay ${ }^{\circledR}$ which was not sensitive enough to allow quantitative analyses.

Interestingly, Himmerich et al. (2016) were the first to investigate the effect of psychiatric therapy and specifically inpatient psychotherapy including EMDR on serum levels of TNF- $\alpha$ in a sample of German soldiers suffering from PTSD. Their findings showed that psychotherapeutic management of PTSD is associated with significant changes in the TNF- $\alpha$ system.

\section{PTSD and BDNF}

Brain-derived neurotrophic factor (BDNF) is an important neurotrophin, involved in neurogenesis and neural plasticity of the brain. In addition to brain tissue, BDNF is also present in plasma, serum, whole blood and blood cells. Brain tissue is the largest source of circulating BDNF, contributing to as much as $70-80 \%$ of plasma BDNF (Homberg et al. 2014).

Stress has been widely linked to abnormal BDNF homeostasis, which, in turn, plays a negative role in the pathophysiology of several psychiatric disorders, including PTSD (Kelmendi et al. 2016) and depression (Karlović et al. 2013). Low BDNF levels are also proposed to be a biological marker of suicidal behavior in the general and in certain populations of psychiatric patients (Sher 2011). Unlike in major depressive disorder (MDD), contrasting findings have been reported regarding peripheral levels of BDNF in patients with PTSD.

In patients with PTSD, peripheral BDNF levels were decreased in patients with different types of non-combat related trauma (Dell'Osso et al. 2009) and L'Aquilla earthquake survivors, unchanged in subjects with traffic accidents ( $\mathrm{Su}$ et al. 2015, van den Heuvel et al. 2016), and even increased in subjects severely injured in motor vehicle accidents (Matsuoka et al. 2013) and with nonspecified trauma (Hauck et al. 2010). It was also reported that BDNF is higher early after trauma, and decreases thereafter (Hauck et al. 2010). Peripheral BDNF levels were positively correlated with impulsiveness in 23 Italian PTSD patients with no specified trauma (Martinotti et al. 2015). However, baseline serum BDNF levels were not correlated with the severity of PTSD symptoms in a small sample of 16 veterans and civilians (Berger et al. 2010). In addition, 16 patients with civilian PTSD due to physical and/or sexual abuse or motor vehicle accidents had no change in cerebrospinal fluid (CSF) BDNF levels, compared to nontraumatized healthy controls (Bonne et al. 2011). 
In the Framingham Study, low serum BDNF levels were associated with increased risk of stroke (Pikula et al. 2013) and plasma BDNF levels were low in patients with heart failure (Takashio et al. 2015). It was hypothesized that BDNF can inhibit inflammation (Singh et al. 2014). Decreased BDNF levels have been proposed as a risk factor for CVD, obesity, diabetes (Singh et al. 2014) and as a biomarker of heart failure (Takashio et al. 2015). Conversely, higher serum levels are associated with a decreased risk of CVD and mortality (Kaess et al. 2015).

In spite of extensive investigation of peripheral BDNF levels in PTSD related to different types of traumatic events, there is no data on BDNF levels in war veterans. Unlike PTSD, after natural disasters and traffic accidents, which are associated with single events, combat-related PTSD is related to numerous traumatic events during a prolonged period of time. Although not separated in current diagnostic classifications, combatrelated and civilian PTSD might have somewhat different clinical presentation, severity, and biological background. Therefore, findings from the studies on subjects with non-combat related PTSD might not generalize to war veterans suffering from PTSD. For example, animal studies suggest that surviving more than one traumatic event (i.e., multiple hits) might be a critical contributor to the development of PTSD (Finsterwald et al. 2015). In addition, a higher total number of traumatic experiences during the war in Croatia was a significant predictor of all three clusters of PTSD symptoms (Lončar et al. 2014).

\section{Quantitative electroencephalogram (qEEG)}

Quantitative electroencephalogram (qEEG) has revealed several abnormalities in patients with PTSD, such as changes in EEG rhythm (Begić et al. 2001) and asymmetry (Meyer et al. 2015). Clinical experience has indicated that particular symptom clusters differently influence qEEG. Hyperarousal symptoms have been associated with increased sympathetic activity, which might influence EEG in terms of increasing $\beta$ rhythm and frontal asymmetry. While chronic autonomic activation is proposed to be one among several links between PTSD and increased CV risk (Kibler et al. 2014), the association between qEEG and CV risk factors remains unknown and further empirical studies are warranted.

\section{Psychological factors}

In addition to the biological factors, some psychological characteristics of individuals suffering from PTSD are believed to contribute to the development of CVD. Personality traits, conceptualized as dimensions of individual differences in tendencies to show consistent patterns of thoughts, feelings, and actions across developmental periods and contexts (McCrae \& Costa 2003), play an important role in the development and symptomatic expressions of PTSD (DiGangi et al. 2013,
Jakšić et al. 2012). PTSD severity is consistently related to trait neuroticism/harm avoidance (i.e., a tendency to experience negative mood and react with high anxiety and worry to adverse events) and trait impulsivity/ hostility (DiGangi et al. 2013, Jakšić et al. 2012, Orth \& Wieland 2006), independent of other factors such as age, early-life trauma and frequency of war-related traumatic events (Stevanović et al. 2016). It seems personality traits might lead to increased CVD risks through the augmentation of PTSD symptom severity.

Significant links were reported between personality traits and cardiovascular distress in the general population. Higher levels of trait anger and hostility were prospectively associated with angina pectoris, myocardial infarction, and angiographic severity of coronary heart disease among older community-dwelling men (Kawachi et al. 1996). Adult male inpatients who had experienced acute myocardial infarction exhibited increased trait impulsivity and trait anxiety when compared to a healthy control group (Milicic et al. 2016). In recent years, the concept of type-D personality was formulated by Denollet (2005), in response to the findings that depression and low perceived social support are related to cardiovascular morbidity and mortality. He proposed a personality type that might predispose to depression and social isolation by combining two personality traits, namely negative affectivity (the tendency to experience negative emotions) and social inhibition (the tendency to inhibit self expression in social interactions). Type-D personality has been shown to predict adverse clinical outcomes in patients after acute coronary syndrome and revascularisation, and in patients with chronic heart failure (Denollet et al. 2006, Pedersen \& Denollet 2003). It is also associated with heightened proinflammatory cytokine activation in patients with heart failure (Denollet et al. 2003), and with disturbances in cortisol secretion after acute coronary syndrome (Whitehead et al. 2007). More recently, typeD personality was associated with coronary artery plaque presence and severity in people with no history of cardiovascular disease, even after adjustment for typical cardiac risk factors (Compare et al. 2014).

Plausible pathophysiological pathways linking personality to cardiovascular complications and adverse clinical outcomes, might be related to hypothalamicpituitary-adrenal-axis hyperreactivity, autonomic and inflammatory dysregulation, and increased oxidative stress (Chida \& Steptoe 2009, Denollet \& Conraads 2011). Indeed, a meta-analytic investigation has found a trend toward significance in the association between type-D personality and biomarkers of CVD (O'Dell et al. 2011). For example, there is some evidence that individuals with high neuroticism have a blunted cortisol response to stress, reflecting down-regulation of the hypothalamic-pituitary-adrenal (HPA) axis due to prolonged cortisol elevation (Lahey 2009). Neuroticism was also associated with higher leukocyte counts (Lahey 2009), and leukocyte count is considered important as a 
potential indicator of inflammation and chronic infection, and is a predictor of mortality from all causes, particularly from CVD (Margolis et al. 2005). Additionally, a relationship between personality and cardiovascular morbidity could be partially explained by particular behavioral mechanisms. One study found that patients with type-D personality with chronic heart failure were less likely to report cardiac symptoms such as swollen legs and feet or shortness of breath to clinical staff (Schiffer et al. 2007), as a consequence of higher levels of social inhibition (i.e. type-D personality trait). However, type-D personality did not predict the development of PTSD in a prospective study on Dutch soldiers (Rademaker et al. 2011). Unlike social inhibition, negative affectivity independently predicted PTSD symptoms (Rademaker et al. 2011), which further suggested the role of neuroticism in PTSD. While type-D personality might not predict PTSD, it is still unknown whether it contributes to CVD risk in patients who already have PTSD.

To the best of our knowledge, no prior research has simultaneously investigated the role of CVD risk factors and personality traits in predicting cardiac events among individuals suffering from PTSD. There is a lack of empirical data on complex interrelations between somatic CVD risk factors, personality traits, and cardiac outcomes in the PTSD population, despite the evidence obtained from community settings and from those treated for coronary heart disease. Considering an increasing understanding of neurobiological underpinnings of human personality traits, including their associations with systemic inflammation (e.g., CRP and IL-6) and BDNF (Luchetti et al. 2014), future empirical studies on personality-cardiovascular risk factors relationship are necessary. This could lead to more specific screening methods in detecting PTSD patients with a particularly high risk for developing CVD. Finally, in addition to reducing the CVD risk by ameliorating specific symptoms of PTSD with standardized traumafocused psychotherapeutic methods, it might also be further reduced through beneficial effect of antidepressant medications (e.g. paroxetine) on personality traits (Tang et al. 2009).

\section{Behavioral factors}

The relationship between PTSD and increased risk for CVD can be enhanced by unhealthy lifestyles. These, in turn, are dependent on individual's personality traits, particularly the abovementioned ones: neuroticism/harm avoidance and impulsiveness/hostility. Most notably, high prevalence of CV disorders in patients with PTSD might be related to increased frequency of cigarette smoking, with some reports suggesting up to $86 \%$ smoking rates in clinical PTSD samples (Kelly et al. 2015). Smoking behavior might also differ in patients with PTSD, in terms of more difficult smoking cessation and stronger withdrawal symptoms (Kelly et al. 2015). Similar findings have been reported on the alcohol and other toxic substances' abuse in this population, also linking it to self-medication of anxiety and hyperarousal symptoms (Edmondson \& Cohen 2013). In addition, a recent meta-analysis has confirmed an association between PTSD and increased BMI (Suliman et al. 2016). Here, decreased physical activity and unhealthy eating habits in patients with PTSD seem to be related to obesity (Hall et al. 2015).

There is preliminary evidence that changes in physical activity or diet may decrease PTSD and depressive symptoms among people with PTSD (Hall et al. 2015, Rosenbaum et al. 2015). These targeted interventions could prove to be highly useful because sedentary behavior is strongly connected to other cardiac risk factors, such as increased blood pressure, insulin resistance, and high cholesterol levels (Brock et al. 2005). Once again, an indirect effect of personality traits on the increased risk of CVD in PTSD populations could in part be reflected in unhealthy life habits of these individuals. For example, previous studies have linked alcohol/substance use and unhealthy emotional eating to trait neuroticism/harm avoidance and impulsivity in general population (Dick et al. 2010, Keller \& Siegrist 2015). More research is needed to uncover specific relations, including potential mediating and moderating factors, among biological, psychological and behavioral risk factors for CVD in patients with PTSD.

\section{PTSD AND CVDS FROM THE MIND-BODY MEDICINE PERSPECTIVE: RESILIENCE TYING THE MIND, BRAIN AND HEART TOGETHER}

Resilience is increasingly recognized as an important determinant for better understanding and successful treatment of PTSD and CVDs. Resilience may be defined as a collection of protective factors that mediate the relationship between a traumatic stress, disease and positive outcomes. Individuals may be resilient in one domain and not in others, or they may be resilient at one time period and not at other periods of their lives. Resilience enables an individual not only to adapt but also to be better off and to grow in addition to overcoming a specific adversity. Patients with low resilience scores have less ability to deal with traumatic stress and other challenges related to the process of becoming ill (Cal et al. 2015). Both stress and resilience have been related to psychophysiological processes that may influence on both PTSD and CVDs. Strategies to enhance resilience fostering coherence, collaboration, competence, and confidence are investigated and developed aiming to improve prognosis in patients with PTSD (Connor 2006) and CVDs (Kralik et al. 2006, Lemos et al. 2015, Nabi \& Kan 2017).

According to the PASTOR (the positive appraisal style theory of resilience), diminished resilience on exposure to traumatic stress is the key pathophysiological mechanism in PTSD (Kalisch et al. 2015). Quite 
a number of neurobiological papers (Dudley et al. 2011, Franklin et al. 2012, Russo et al 2012, Pfau \& Russo 2015, James et al. 2015) have enumerated long list of epigenetic mechanisms, molecules or brain systems (glutamate receptors in hippocampus, $\triangle \mathrm{FosB}$, FKBP5 polymorphism, apoE gene, dehydroepiandrosterone (DHEA), oxytocin, brain derived neurotrophic factor (BDNF), or serotonin, HHA axis, the brain reward system, the neural circuitry of fear, the appraisal system, etc.) which are associated with resilience, PTSD and CVDs. Given the role of the above mentioned biological factors in cardiovascular disorders, the intricate connections between PTSD and increased CVD risk are even more evident. The worldwide trends have focused on promoting and maintaining resilience, well-being and health rather than providing secondary treatment (Nabi \& Khan 2017).

\section{Resilience and the heart}

There is a growing body of evidence showing that assertive behavior, positive emotions, cognitions and personality traits have been associated with better cardiovascular health. Resilient persons use positive emotions and cognitions to rebound from traumatic experiences effectively and find positive meaning in adversities. According to Arrebola-Moreno et al. (2014) self-efficacy - a component of resilience - was negatively related to both myoglobin and troponin after the acute coronary event suggesting that resilience decreases the extent of the myocardial infarction by affecting the inflammatory response and so showing a protective effect. Cardiac vagal tone and resilience interact synergistically in the promotion of stress recovery (Souza et al. 2007). It is presumed that positive emotions and cognitions facilitate resilience to acute and chronic stress as well as that greater vagal tone is associated with more endurance under stressful and traumatic situations. Some research indicates that improved resilience in cardiovascular patients resulted in their higher satisfaction and quality of life (Nouri-Saed et al. 2015).

\section{Resilience and the brain}

Allostasis is determined by the activity of the CNS. Brain is the most important organ for determining what is perceived as stressful, dangerous and harmful, but it is also a target organ of stress. Resilience is an example of successful allostasis in which wear and tear is minimized (Logan \& Barksdale 2008). Traumatic stress stimulates CNS and hypothalamic pituitary track which leads to allostatic overload, central obesity, insulin resistance, inflammation, arterial hypertension, platelet activation, and endocrine dysfunction leading to the development of CVDs (Logan 2008, Nabi \& Khan 2017). According to some authors, DHEA, neuropeptide $\mathrm{Y}$, galanin, serotonin, oxytocin, testosterone, estrogen, and benzodiazepine receptors are resilience promoting molecules, while the release of corticotropin-releasing hormone (CRH) and cortisol as well as the locus ceruleus norepinephrine system may tend to undermine resilience (Charney 2004, Yehuda et al. 2006, van Zuiden et al. 2017). Interestingly enough low DHEA/ cortisol ratio was proposed as useful biological resilience marker in patients with PTSD. According to Polyvagal Theory (Porges 2007), modulation of vagal tone is very important mode through which the brain circuits regulate the stress responses and quick recovery from traumatic effects.

\section{Resilience and the mind}

The perception of brain and appraisal of events are influenced by personal frame of reference related to personality structure and dynamics involving genes, individual experiences, early development and learned behaviors. It is well known that if patients with PTSD are able to find a purpose, or a reason for which something should be done, and set themselves to do this task, they will be able to achieve personal recovery through the power of resilience. The hardy-resilient style, which distinguishes healthy stress responders from unhealthy ones, is associated with higher highdensity lipoprotein cholesterol levels (Bartone et al. 2008). The hard-resilient style involves 1 . a strong sense of commitment, a belief that individual can control or influence outcomes; 2. an exploring approach to living, a sense of challenge; and 3. a strong future orientation, living at the present and learning from the past; 4. a sense of humor and optimism. Some studies have observed that the type-D personality, which is a risk factor for CVD, was positively correlated with PTSD symptoms, and negatively correlated with resilience (Cho \& Kang 2017).

\section{CONCLUSION}

There are several biological, psychological and behavioral mechanisms which contribute to CVD morbidity and mortality in patients with PTSD. Relationships among all these factors are complex and yet incompletely understood. Resilience is the capacity for and process of successful overcoming traumatic stress. Given a high prevalence of CVD in patients with PTSD, there is an urgent need for a more active monitoring, treatment (Beristianos et al. 2016), increasing resilience and multidisciplinary management (Jakovljević et al. 2012, Rosenbaum et al. 2015) of PTSD patients, particularly war veterans. Potential biomarkers, as well as some personality and behavioral characteristics, may be useful for assessing both the CVD risks and the utility of potential interventions and resilience training. We believe that a thorough monitoring and treatment of generally recognized main CVD risk factors might significantly decrease cardiovascular morbidity and mortality in this particularly vulnerable population. 


\section{Acknowledgements: None.}

\section{Conflict of interest: None to declare.}

\section{Contribution of individual authors:}

Marina Šagud: research idea, literature search, first manuscript draft, manuscript revisions, approval of the final version;

Nenad Jakšić: literature search, first manuscript draft, manuscript revisions, approval of the final version;

Bjanka Vuksan Ćusa: literature search, first manuscript draft, manuscript revisions, approval of the final version;

Mladen Lončar: manuscript revisions, approval of the final version;

Ivana Lončar: manuscript revisions, approval of the final version;

Alma Mihaljević Peleš: first manuscript draft, manuscript revisions, approval of the final version;

Davor Miličić: literature search, first manuscript draft, manuscript revisions, approval of the final version;

Miro Jakovljević: literature search, first manuscript draft, manuscript revisions, approval of the final version.

\section{References}

1. Arrebola-Moreno AL, Garcia-Retamero T, Catena A, Marfil-Alvarez $R$, Melgares-Moreno $R$ \& Ramirez-Hernandez JA: On the protective effct of resilience in patients with acute coronary syndrome. International Journal of Clinical and Health psychology 2014; 14:111-119.

2. Barron E, Lara J, White M, Mathers JC: Blood-borne biomarkers of mortality risk: Systematic review of cohort studies. PLoS One 2015; 10:1-23.

3. Bartone PT, Spinosa T, Robb J \& Pastel RH: Hardyresilient style is associated with high-density lipoprotein levels. Presented at the Association of Military surgeons of the United States annual meeting, San Antonio, Texas, 11 November, 2008

4. Begic D, Hotujac L, Jokic Begic N: Electroencephalographic comparison of veterans with combat-related posttraumatic stress disorder and healthy subjects. Int $J$ Psychophysiol 2001; 40:167-72.

5. Berger W, Mehra A, Lenoci M, Metzler TJ, Otte C, Tarasovsky G, et al: Serum brain-derived neurotrophic factor predicts responses to escitalopram in chronic posttraumatic stress disorder. Prog Neuropsychopharmacol Biol Psychiatry 2010; 34:1279-84.

6. Beristianos MH, Yaffe K, Cohen B, Byers AL: PTSD and Risk of Incident Cardiovascular Disease in Aging Veterans. Am J Geriatr Psychiatry 2016; 24:192-200.

7. Bogic M, Ajdukovic D, Bremner S, Franciskovic T, Galeazzi GM, Kucukalic A, et al: Factors associated with mental disorders in long-settled war refugees: Refugees from the former Yugoslavia in Germany, Italy and the UK. Br J Psychiatry 2012; 200:216-23.

8. Bonne O, Gill JM, Luckenbaugh DA, Collins C, Owens MJ, Alesci S, Vythilingam M: Corticotropin-releasing factor, interleukin-6, brain-derived neurotrophic factor, insulin-like growth factor-1, and substance $P$ in the cerebrospinal fluid of civilians with posttraumatic stress disorder before and after treatment with paroxetine. Journal of Clinical Psychiatry 2011; 72:1124-1128.

9. Boscarino JA: Posttraumatic stress disorder and mortality among U.S. Army veterans 30 years after military service. Ann Epidemiol 2006; 16:248-56.

10. Boscarino JA: A prospective study of PTSD and early-age heart disease mortality among Vietnam veterans: implications for surveillance and prevention. Psychosomatic Medicine 2008; 70:668-76.

11. Britvić D, Antičević $V$, Kaliterna M, Lušić L, Beg A, Brajević-Gizdić I, et al: Comorbidities with Posttraumatic Stress Disorder (PTSD) among combat veterans: 15 years postwar analysis. Int J Clin Heal Psychol 2015; 15:81-92.

12. Brock CM, Pharm D, King DS, Pharm D, Wofford MR, Harrell TK, et al: Exercise, Insulin Resistance, and Hypertension: A Complex Relationship. Metabolic Syndrome and Related Disorders 2005; 3:60-65.

13. Cal SF, de Sa LB, Glustak ME \& Santiago MB: Resilience in chronic disease: A systematic review. Cogent Psychology 2015; 2.1024928.http:

//dx.doi.org/10.1080/23311908.2015.1024928

14. Canetti D, Russ E, Luborsky J, Gerhart JI, Hobfoll SE: Inflamed by the flames? The impact of terrorism and war on immunity. J Trauma Stress 2014; 27:345-52.

15. Charney DS: Psychobiological mechanisms of resilience and vulnerability: Implications for successful adaptation to extreme stress. Am J Psychiatry 2004; 161:195-216.

16. Chida Y, Steptoe A: The Association of Anger and Hostility With Future Coronary Heart Disease. A Meta-Analytic Review of Prospective Evidence. J Am Coll Cardiol 2009; 53:936-46.

17. Cho GJ \& Kang J: Type D personality and post-traumatic stress disorder symptoms among intensive care unit nurses: The mediating effect of resilience. PLOS ONE 2017; 12:e0175067.

18. Compare A, Mommersteeg PMC, Faletra F, Grossi E, Pasotti E, Moccetti T, Auricchio A: Personality traits, cardiac risk factors, and their association with presence and severity of coronary artery plaque in people with no history of cardiovascular disease. Journal of Cardiovascular Medicine 2014; 15:423-30.

19. Connor KM: Assessment of resilience in the aftermath of trauma. J Clin Psychiatry 2006; 67(suppl 2):46-49.

20. de Vries GJ, Lok A, Mocking R, Assies J, Schene A, Olff M: Altered one-carbon metabolism in posttraumatic stress disorder. J Affect Disord 2015; 184:277-85.

21. Dell'Osso L, Carmassi C, Del Debbio A, Dell'Osso MC, Bianchi C, da Pozzo E, et al: Brain-derived neurotrophic factor plasma levels in patients suffering from posttraumatic stress disorder. Prog Neuro-Psychopharmacology Biol Psychiatry 2009; 33:899-902.

22. Denollet J: DS14: Standard Assessment of Negative Affectivity, Social Inhibition, and Type D Personality. Psychosom Med 2005; 67:89-97.

23. Denollet J, Conraads VM, Brutsaert DL, De Clerck LS, Stevens WJ, Vrints CJ: Cytokines and immune activation in systolic heart failure: The role of Type D personality. Brain Behav Immun 2003; 17:304-9.

24. Denollet J, Pedersen SS, Ong ATL, Erdman RAM, Serruys $P W$, Van Domburg RT: Social inhibition modulates the effect of negative emotions on cardiac prognosis following 
percutaneous coronary intervention in the drug-eluting stent era. Eur Heart J 2006; 27:171-7.

25. Denollet J, Conraads VM: Type D personality and vulnerability to adverse outcomes in heart disease. Cleveland Clinic Journal of Medicine 2011; 78:S13-9.

26. Dick DM, Smith G, Olausson P, Mitchell SH, Robert F, Malley SSO, et al: Alcohol Use Disorders. Addict Biol 2010; 15:217-26.

27. DiGangi JA, Gomez D, Mendoza L, Jason LA, Keys CB, Koenen KC: Pretrauma risk factors for posttraumatic stress disorder: A systematic review of the literature. Clin Psychol Rev 2013; 33:728-44.

28. Dudley KJ, Li X, Kobor MS, Kippin TE \& Bredy TW: Epigenetic mechanisms mediating vulnerability and resilience to psychiatric disorders. Neuroscience and Biobehavioral Reviews 2011; 35:1544-1551.

29. Edmondson D, Cohen BE: Posttraumatic Stress Disorder and Cardiovascular Disease. Prog Cardiovasc Dis 2013; 55:548-56.

30. Farr OM, Ko BJ, Joung KE, Zaichenko L, Usher N, Tsoukas M, et al: Posttraumatic stress disorder, alone or additively with early life adversity, is associated with obesity and cardiometabolic risk. Nutr Metab Cardiovasc Dis 2015; 25:479-88.

31. Feder A, Nestler EJ \& Charney DS: Psychobiology and molecular genetics of resilience. Nat Rev Neurosci 2009; 10:446-457.

32. Finsterwald C, Steinmetz AB, Travaglia A, Alberini CM: From memory impairment to posttraumatic stress disorder-like phenotypes: The critical role of an unpredictable second traumatic experience. J Neurosci 2015; 35:15903-15.

33. Franklin TB, Saab BJ \& Mansuy IM: Neural mechanism of stress resilience and vulnerability. Neuron 2012; 75:747-761.

34. Galasko GIW, Lahiri A, Barnes SC, Collinson P, Senior $R$ : What is the normal range for $N$-terminal pro-brain natriuretic peptide? How well does this normal range screen for cardiovascular disease? Eur Heart J 2005; 26:2269-76.

35. Gates MA, Holowka DW, Vasterling JJ, Keane TM, Marx $B P$, Rosen RC: Posttraumatic stress disorder in veterans and military personnel: epidemiology, screening, and case recognition. Psychol Serv 2012; 9:361-82.

36. Gola H, Engler H, Schauer M, Adenauer H, Riether C, Kolassa S, et al: Victims of rape show increased cortisol responses to trauma reminders: A study in individuals with war- and torture-related PTSD. Psychoneuroendocrinology 2012; 37:213-20.

37. Goldstein RB, Smith SM, Chou SP, Saha TD, Jung J, Zhang H, et al: The epidemiology of DSM-5 posttraumatic stress disorder in the United States: results from the National Epidemiologic Survey on Alcohol and Related Conditions-III. Soc Psychiatry Psychiatr Epidemiol 2016; 51:1137-48.

38. Gradus JL, Farkas DK, Svensson E, Ehrenstein V, Lash $T L$, Milstein A, et al: Associations between stress disorders and cardiovascular disease events in the Danish population. BMJ Open 2015; 5:e009334.

39. Grenon SM, Owens CD, Alley H, Perez S, Whooley MA, Neylan TC, et al: Posttraumatic Stress Disorder Is Associated With Worse Endothelial Function Among Veterans. J Am Heart Assoc 2016; 5:1-7.
40. Guo X, Meng Z, Huang G, Fan J, Zhou W, Ling $W$, et al: Meta-analysis of the prevalence of anxiety disorders in mainland China from 2000 to 2015. Sci Rep 2016; 6:28033.

41. Hall KS, Hoerster KD, Yancy WS: Post-traumatic stress disorder, physical activity, and eating behaviors. Epidemiol Rev 2015; 37:103-15.

42. Hauck S, Kapczinski F, Roesler R, de Moura Silveira E, Magalhães PV, Kruel LRP, et al: Serum brain-derived neurotrophic factor in patients with trauma psychopathology. Prog Neuro-Psychopharmacology Biol Psychiatry 2010; 34:459-62.

43. Heppner PS, Crawford EF, Haji U, Afari N, Hauger RL, Dashevsky $B$ a, et al: The association of posttraumatic stress disorder and metabolic syndrome: a study of increased health risk in veterans. BMC Med 2009; 7:1.

44. Himmerich H, Willmund GD, Zimmermann P, Wolf JE, Buehler AH, Kirkby KC, Dalton B, Holdt LM, Teupser D, Wesemann U: Serum concentrations of TNF-alpha and its soluble receptors during psychotherapy in German soldiers suffering from combat-related PTSD. Psychiatr Danub 2016; 28:293-8.

45. Homberg JR, Molteni R, Calabrese F, Riva MA: The serotonin-BDNF duo: Developmental implications for the vulnerability to psychopathology. Neurosci Biobehav Rev 2014; 43:35-47.

46. Jakovljević M, Brajković L, Jakšić N, Lončar M, Aukst Margetić B \& Lasić D: Posttraumatic stress disorders (PTSD) from different perspectives: A transdisciplinary integrative approach. Psychiatr Danub 2012; 24:246-55.

47. Jakšić N, Brajković L, Ivezić E, Topić $R$ \& Jakovljević M: The role of personality traits in posttraumatic stress disorder (PTSD). Psychiatr Danub 2012; 24:256-266.

48. James LM, Engdahl BE \& Georgopoulous AP: Apolipoprotein E: the resilience gene. Exp Brain Res 2017; 235:1853-1859.

49. Jendricko T, Vidović A, Grubisić-Ilić M, Romić Z, Kovacić Z, Kozarić-Kovacić D: Homocysteine and serum lipids concentration in male war veterans with posttraumatic stress disorder. Prog Neuropsychopharmacol Biol Psychiatry 2009; 33:134-40.

50. Jergovic M, Bendelja K, Mlakar AS, Vojvoda V, Aberle N, Jovanovic T, et al: Circulating levels of hormones, lipids, and immune mediators in posttraumatic stress disorder - a three-month follow-up study. Front Psychiatry 2015; 6:1-13.

51. Jergović M, Tomičević M, Vidović A, Bendelja K, Savić A, Vojvoda $V$, et al: Telomere shortening and immune activity in war veterans with posttraumatic stress disorder. Prog Neuropsychopharmacol Biol Psychiatry 2014; 54:275-83.

52. Kadojic D, Demarin V, Kadojic M, Mihaljevic I, Barac B: Influence of prolonged stress on cerebral hemodynamics. Coll Antropol 1999; 23:665-72.

53. Kaess BM, Preis SR, Lieb W, Beiser AS, Yang Q, Chen $T C$, et al: Circulating brain-derived neurotrophic factor concentrations and the risk of cardiovascular disease in the community. J Am Heart Assoc 2015; 4:e001544.

54. Kalisch RM, Mueller MB, Tuescher O: A conceptual framework for the neurobiological study of resilience. Behavioral and Brain Sciences 2015; 38:e92.

55. Karlovic D, Serretti A, Jevtovic S, Vrkic N, Seric V, Peles AM: Diagnostic accuracy of serum brain derived neurotrophic factor concentration in antidepressant naive 
patients with first major depression episode. J Psychiatr Res 2013; 47:162-7.

56. Kawachi I, Sparrow D, Spiro A 3rd, Vokonas P, Weiss ST: A prospective study of anger and coronary heart disease. The Normative Aging Study. Circulation 1996; 94:2090 2095.

57. Keller C, Siegrist M: Does personality influence eating styles and food choices? Direct and indirect effects. Appetite 2015; 84:128-38.

58. Kelly MM, Jensen KP, Sofuoglu M: Co-occurring tobacco use and posttraumatic stress disorder: Smoking cessation treatment implications. Am J Addict 2015; 24:695-704.

59. Kelmendi B, Adams TG, Yarnell S, Abdallah CG, Krystal $J H$, Kelmendi B, et al: treatments. PTSD: from neurobiology to pharmacological treatments. European Journal of Psychotraumatology 2016; 7:31858.

60. Kessler RC, Chiu WT, Demler O, Walters EE: Prevalence, severity, and comorbidity of 12-month DSM-IV disorders in the National Comorbidity Survey Replication. Arch Gen Psychiatry 2005; 62:617-27.

61. Kibler JL, Tursich M, Ma M, Malcolm L, Greenbarg R: Metabolic, autonomic and immune markers for cardiovascular disease in posttraumatic stress disorder. World J Cardiol 2014; 6:455-61.

62. Kralik D, van Loon \& Visentin $K$ : resilience in chronic illness experience. Educational Action Research 2006; 14:187-201. DOI:10.1080/09650790600718035

63. Lahey BB: Public health significance of neuroticism. Am Psychol 2009; 64:241-56.

64. Lemos CM, Moraes DM \& Pellanda LC: Resilience in patients with ischemic heart disease. Arq Bras Cardiol 2015; (online).ahead print, PP.0-0. DOI: $10.5935 / a b c .20160012$

65. Levine J, Timinsky I, Vishne T, Dwolatzky T, Roitman S, Kaplan Z, et al: Elevated serum homocysteine levels in male patients with PTSD. Depress Anxiety 2008; 25:154-7.

66. Logan JG \& Barksdale DJ: Allostasis and allostatic load: expanding the discourse on stress and cardiovascular disease. Journal of Nursing and Healthcare of Chronic Illness in association with Journal of Clinical Nursing 2008; 17:201-208.doi:10.1111/j.13652702.2008.02347.x

67. Lončar M, Plašć ID, Bunjevac T, Hrabač P, Jakšić N, Kozina S, Marčinko D: Predicting symptom clusters of posttraumatic stress disorder (PTSD) in Croatian war veterans: the role of socio-demographics, war experiences and subjective quality of life. Psychiatr Danub 2014; 26:231-8.

68. Loppnow H, Buerke M, Werdan K, Rose-John S: Contribution of vascular cell-derived cytokines to innate and inflammatory pathways in atherogenesis. J Cell Mol Med 2011; 15:484-500.

69. Margolis KL, Manson JE, Greenland P, Rodabough RJ, Bray PF, Safford M, Prentice R: Leukocyte count as a predictor of cardiovascular events and mortality in postmenopausal women: The Women's Health Initiative Observational Study. Archives of Internal Medicine 2005; 165:500-508.

70. Marksteiner J, Imarhiagbe D, Defrancesco M, Deisenhammer EA, Kemmler G, Humpel C: Analysis of 27 vascular-related proteins reveals that NT-proBNP is a potential biomarker for Alzheimer's disease and mild cognitive impairment: A pilot-study. Exp Gerontol 2013; 50:114-21.
71. Martinotti G, Sepede G, Brunetti M, Ricci V, Gambi F, Chillemi E, et al: BDNF concentration and impulsiveness level in post-traumatic stress disorder. Psychiatry Res 2015; 229:814-8.

72. Matsuoka Y, Nishi D, Noguchi H, Kim Y, Hashimoto K: Longitudinal changes in serum brain-derived neurotrophic factor in accident survivors with posttraumatic stress disorder. Neuropsychobiology 2013; 68:44-50.

73. Meyer T, Smeets T, Giesbrecht T, Quaedflieg CWEM, Smulders FTY, Meijer EH, et al: The role of frontal EEG asymmetry in post-traumatic stress disorder. Biol Psychol 2015; 108:62-77.

74. Michaud M, Balardy L, Moulis G, Gaudin C, Peyrot C, Vellas B, et al: Proinflammatory cytokines, aging, and agerelated diseases. J Am Med Dir Assoc 2013; 14:877-82.

75. Michopoulos V, Rothbaum AO, Jovanovic T, Almli LM, Bradley B, Rothbaum BO, et al: CRP genetic variation and CRP levels are associated with increased PTSD symptoms and physiological responses in a highly traumatized civilian population. Am J Psychiatry 2015; 172:353-62.

76. Milicic D, Brajkovic L, Macek JL, Andric A, Ardalic Z, Buratovic T \& Marčinko D: Type A Personality, Stress, Anxiety and Health Locus of Control in Patients with Acute Myocardial Infarction. Psychiatr Danub 2016; 28:409-414.

77. Mirza SS, de Bruijn RFG, Koudstaal PJ, van den Meiracker AH, Franco OH, Hofman A, et al: The $N$ terminal pro B-type natriuretic peptide, and risk of dementia and cognitive decline: a 10-year follow-up study in the general population. J Neurol Neurosurg Psychiatry 2015; 87:356-62.57.

78. Nabi AU \& Khan MI: Resilience as predictor of mental well-being among cardiovascular disorder. The International Journal of Indian Psychology 2017; 4:182-190.

79. Nouri-Saeed A, Salari A, Nouri-Saeed A, Rouhi-Balasi L $\&$ Moaddab F: Resilience and ssociated factors in patients with coronary artery disease. Journal of Nursing and Midwifery Sciences 2015; 2:23-28.

80. O'Dell KR, Masters KS, Spielmans GI, Maisto SA: Does type-D personality predict outcomes among patients with cardiovascular disease? A meta-analytic review. J Psychosom Res 2011; 71:199-206.

81. Orth U, Wieland E: Anger, hostility, and posttraumatic stress disorder in trauma-exposed adults: A meta-analysis. J Consult Clin Psychol 2006; 74:698-706.

82. Pacella ML, Hruska B, Delahanty DL: The physical health consequences of PTSD and PTSD symptoms: A metaanalytic review. J Anxiety Disord 2013; 27:33-46.

83. Passos IC, Vasconcelos-Moreno MP, Costa LG, Kunz M, Brietzke E, Quevedo J, et al: Inflammatory markers in post-traumatic stress disorder: A systematic review, metaanalysis, and meta-regression. The Lancet Psychiatry $2015 ; 2: 1002-12$.

84. Pedersen SS, Denollet J: Type D personality, cardiac events, and impaired quality of life: a review. Eur $J$ Cardiovasc Prev Rehabil 2003; 10:241-8.

85. Pfau ML \& Russo SJ: Peripheral and central mechanisms of stress relilience. Neurobiology of Stress 2015; 1:66-79.

86. Pikula A, Beiser AS, Chen TC, Preis SR, Vorgias D, Decarli $C$, et al: Serum brain-derived neurotrophic factor and vascular endothelial growth factor levels are associated with risk of stroke and vascular brain injury: Framingham study. Stroke 2013; 44:2768-75. 
87. Porges SW: The polyvagal perspective. Biol Psychology 2007; 74:116-143.

88. Priebe S, Bogic M, Ajdukovic D, Franciskovic T, Galeazzi GM, Kucukalic A, et al: Mental Disorders Following War in the Balkans. Arch Gen Psychiatry 2010; 67:518.

89. Rademaker AR, van Zuiden M, Vermetten E, Geuze E: Type D Personality and the Development of PTSD Symptoms: A Prospective Study. J Abnorm Psychol 2011; 120:299-307.

90. Rosenbaum S, Vancampfort D, Steel Z, Newby J, Ward $P B$, Stubbs B: Physical activity in the treatment of Posttraumatic stress disorder: A systematic review and metaanalysis. Psychiatry Res 2015; 230:130-6.

91. Russo SJ, Murrough JW, Han MH, Charney DS \& Nestler EJ: Neurobiology of resilience. Nat Neurosci 2012, 15:1475-1484.

92. Schiffer AA, Denollet J, Widdershoven JW, Hendriks EH, Smith ORF: Failure to consult for symptoms of heart failure in patients with a type-D personality. Heart 2007; 93:814-8.

93. Sher L: Brain-derived neurotrophic factor and suicidal behavior. QJM 2011; 104:455-8.

94. Sin NL, Graham-Engeland JE, Almeida DM: Daily positive events and inflammation: Findings from the National Study of Daily Experiences. Brain Behav Immun 2015; 4314:130-8.

95. Solter V, Thaller V, Karlovic D, Crnkovic D: Elevated serum lipids in veterans with combat-related chronic posttraumatic stress disorder. Croat Med J 2002; 43:685-9.

96. Souza GGL, Mendonca-de-Souza ACF, Barros EM, Coutinho EFS, Oliveira L, Mendlowicz MV, Figueira I \& Volchan E: Resilience and vagal tone predict cardiac recovery from acute social stress. Stress 2007; 10:368-374

97. Stein MB, Lang AJ, Laffaye C, Satz LE, Lenox RJ, Dresselhaus TR: Relationship of sexual assault history to somatic symptoms and health anxiety in women. Gen Hosp Psychiatry 2004; 26:178-83.

98. Stevanović A, Frančišković T, Vermetten E: Relationship of early-life trauma, war-related trauma, personality traits, and PTSD symptom severity: A retrospective study on female civilian victims of war. Eur J Psychotraumatol 2016; 7:10.3402/ejpt.v7.30964.

99. Su S, Xiao Z, Lin Z, Qiu Y, Jin Y, Wang Z: Plasma brainderived neurotrophic factor levels in patients suffering from post-traumatic stress disorder. Psychiatry Res 2015; 229:365-9.

100. Suliman S, Anthonissen L, Carr J, du Plessis S, Emsley $R$, Hemmings SMJ, et al: Posttraumatic Stress Disorder, Overweight, and Obesity. Harv Rev Psychiatry 2016; 24:271-93.

101. Takashio S, Sugiyama S, Yamamuro M, Takahama H, Hayashi T, Sugano Y, et al: Significance of low plasma levels of brain-derived neurotrophic factor in patients with heart failure. Am J Cardiol 2015; 116:243.

102. Tang TZ, Derubeis RJ, Hollon SD, Amsterdam J, Shelton $R$, et al: Personality Change During Depression Treatment: A Placebo-Controlled Trial. 2009; 66:1322-30.

103. Turner JH, Neylan TC, Schiller NB, Li Y, Cohen BE: Objective Evidence of Myocardial Ischemia in Patients with Posttraumatic Stress Disorder. Biol Psychiatry 2013; 74:861-6.

104. van den Heuvel L, Suliman S, Malan-Müller S, Hemmings $S$, Seedat $S$ : Brain-derived neurotrophic factor (BDNF) Val66Met polymorphism and plasma levels in road traffic accident survivors. Anxiety Stress Coping 2016; 5806:1-33.

105. van Zuiden M, Haverkort SO, Tan Z, Daams J, LA \& Olf M: DHEA and DHEA-S levels in posttraumatic stress disorder: A meta-analytic review. Psychoneuroendocrinology 2017; 84:76-82.

106. Vidovic A, Grubisic-Ilic M, Kozaric-Kovacic D, Gotovac $K$, Rakos I, Markotic A, et al: Exaggerated platelet reactivity to physiological agonists in war veterans with posttraumatic stress disorder. Psychoneuroendocrinology 2011; 36:161-72.

107. Vuksan-Ćusa B, Jakovljević M, Šagud M, Mihaljević Peleš A, Marćinko D, Topić R, et al: Metabolic syndrome and serum homocysteine in patients with bipolar disorder and schizophrenia treated with second generation antipsychotics. Psychiatry Res 2011; 189:21-5.

108. Whitehead DL, Perkins-Porras L, Strike PC, Magid K, Steptoe A: Cortisol awakening response is elevated in acute coronary syndrome patients with type- $D$ personality. J Psychosom Res 2007; 62:419-25.

109. Yehuda R, Brand SR, Golier JA \& Yang RK: Clinical correlates of DHEA associated with post-traumatic stress disorder. Acta Psychiatr Scand 2006; 114:187193.

\section{Correspondence:}

Nenad Jakšić, MA

National Center for Psychotrauma, Department of Psychiatry,

University Hospital Center Zagreb

Kišpatićeva 12, 10000 Zagreb, Croatia

E-mail:nenad_jaksic@yahoo.com 